\title{
REGULARITY OF SOLUTIONS TO AN ABSTRACT INHOMOGENEOUS LINEAR DIFFERENTIAL EQUATION
}

\author{
G. F. WEBB
}

\begin{abstract}
Let $T(t), t>0$, be a strongly continuous semigroup of linear operators on a Banach space $X$ with infinitesimal generator $A$ satisfying $T(t) X \subset D(A)$ for all $t>0$. Let $f$ be a function from $[0, \infty)$ to $X$ of strong bounded variation. It is proved that $u(t)={ }^{\text {def }} T(t) x+\int{ }^{n} T(t-s) f(s) d s$, $x \in X$, is strongly differentiable and satisfies $d u(t) / d t=A u(t)+f(t)$ for all but a countable number of $t>0$.
\end{abstract}

1. Introduction. Let $T(t), t \geqslant 0$, be a strongly continuous semigroup of bounded linear operators on the Banach space $X$ with infinitesimal generator $A$ and let $f$ be an $X$-valued function on [0, $\infty$ ). Our objective is to establish sufficient conditions so that the function

$$
u(t) \stackrel{\text { def }}{=} T(t) x+\int_{0}^{t} T(t-s) f(s) d s, \quad x \in X,
$$

is a strong solution of the inhomogeneous linear differential equation

$$
d u(t) / d t=A u(t)+f(t), \quad u(0)=x .
$$

It is well known that $u(t)$ satisfies (1.2) for $t \geqslant 0$ provided that $x \in D(A)$ and $f$ is continuously differentiable (see $[4$, Theorem 1.19, p. 486] or [5, Theorem 6.5 , p. 135]). It is also well known that $u(t)$ satisfies (1.2) for $t>0$ provided that $x \in X, T(t), t \geqslant 0$, is homomorphic, and $f$ is Hölder continuous (see [4, Theorem 1.27, p. 491] or [5, Theorem 6.7, p. 138]). The theorem which we will prove demonstrates that $u(t)$ satisfies (1.2) under the assumptions that $T(t) X$ $\subset D(A)$ for $t>0$ and $f$ is of strong bounded variation. The main idea of our proof is to show that under our assumptions the integral in (1.1) lies in $D(A)$ and the image of this integral under $A$ may be represented as a Stieltjes integral.

TheOREM. Suppose $T(t) X \subset D(A)$ for all $t>0$ and $f$ is of strong bounded variation on $[0, r]$. For a given $x \in X$ let $u(t)$ be defined on $[0, r]$ by (1.1). Then, $u(t)$ satisfies the following:

Presented to the Society, September 18, 1975; received by the editors August 14, 1975 and, in revised form, December 16, 1975.

AMS (MOS) subject classifications (1970). Primary 47D05; Secondary 34G05.

Key words and phrases. Strongly continuous semigroup, infinitesimal generator, inhomogeneous equation, strong bounded variation. 
$u(t) \in D(A)$ for $t \in(0, r]$ and $A u(t)$ is continuous on $(0, r]$;

$d^{+} u(t) / d t=A u(t)+f(t+)$ for all $t \in(0, r)$ and $d^{+} u(t) / d t$ is continuous from the right on $(0, r)$;

$d^{-} u(t) / d t=A u(t)+f(t-)$ for all $t \in(0, r]$ and $d^{-} u(t) / d t$ is continuous from the left on $(0, r]$;

$d u(t) / d t=A u(t)+f(t)$ for all but a countable number of points in $[0, r]$ and $d u(t) / d t$ is continuous at all but $a$ countable number of points in $[0, r]$.

Before proving our theorem we first state some facts about Banach spacevalued functions of strong bounded variation.

2. Vector-valued functions of strong bounded variation. Suppose $f$ is of strong bounded variation from $[0, r]$ to $X$ (according to the definition of $[3, \mathrm{p}$. 59]). The following properties of $f$ may be proved analogously to the case of real-valued functions of bounded variation (for a discussion of real-valued functions of bounded variation the reader is referred to [9, Chapter 2] or [2, Chapter II]):

$f$ has a right limit at each $t \in[0, r)$, denoted by $f(t+)$, and $f(\cdot+)$ is right continuous on $[0, r)$;

$f$ has a left limit at each $t \in(0, r]$, denoted by $f(t-)$, and $f(\cdot-)$ is left continuous on $(0, r]$;

$f(\cdot-)$ is of strong bounded variation on $[0, r]$ (where for convenience we define $f(0-)=f(0)$ ), and if we define $\nu(t)$ to be the total variation of $f(\cdot-)$ between 0 and $t$, then $\nu$ is nondecreasing and left continuous on $(0, r]$;

$f$ is bounded on $[0, r]$ and continuous at all but a countable number of points in $[0, r]$.

3. Proof of the theorem. We first prove the lemmas below, each of which is under the hypothesis of the theorem. In what follows we will suppose that $M$ 
is a constant such that $|T(t)| \leqslant M$ for $0 \leqslant t \leqslant r$ (see [4, p. 484]) and $\nu$ is defined as in (2.3).

LEMMA 3.1. If $0<t \leqslant r$, then

$$
\begin{gathered}
\int_{0}^{t} T(t-s) f(s) d s \in D(A) \\
A \int_{0}^{t} T(t-s) f(s) d s=\int_{0}^{t} d T(t-s) f(s-) .
\end{gathered}
$$

Proof. Let $0<t \leqslant r$. We observe that $\int_{0}^{t} T(t-s) f(s) d s$, the Riemann integral, exists since the integrand is bounded and continuous almost everywhere by virtue of $(2.1)$ and the continuity properties of $T(t), t \geqslant 0$. The function $T$ from $[0, t]$ to $B(X, X)$ (where $B(X, X)$ denotes the Banach space of bounded linear operators on $X)$ is bounded on $[0, t]$. Further, since $T(t) X \subset D(A)$ for $t>0, T$ is continuous from $(0, t]$ to $B(X, X)$ (see [3, Theorem 10.3.5, p. 310]). By (2.3) the set of discontinuities of $T(t-s)$, considered as a function of $s$ in $[0, t]$ to $B(X, X)$, has $\nu$ measure 0 . That is, $s \rightarrow T(t-s)$ is discontinuous only at $t$ and, by (2.3), $\lim _{s \rightarrow t^{-}} \nu(s)=\nu(t)$. Thus, the Riemann-Stieltjes integral $\int_{0}^{t} d T(t-s) f(s-)$ exists in the sense that for each $\varepsilon>0$ there exists $\delta>0$ such that if $\left\{s_{i}\right\}_{i=0}^{n}$ is a chain from 0 to $t$ such that $\sup _{i=1, \ldots, n}\left|s_{i}-s_{i-1}\right|<\delta$, and $s_{i-1} \leqslant s_{i}^{\prime} \leqslant s_{i}$, then

$$
\left\|\sum_{i=1}^{n}\left(T\left(t-s_{i}\right)-T\left(t-s_{i-1}\right)\right) f\left(s_{i}^{\prime}-\right)-\int_{0}^{t} d T(t-s) f(s-)\right\|<\varepsilon
$$

(see [2, Theorem 13.16, p. 65 and Theorem 11.7, p. 53]).

For each positive integer $n$ let $s_{i}^{n}=i t / n$, where $i=0,1, \ldots, n$. Define $g_{n}$ : $[0, t] \rightarrow X$ by $g_{n}(s)=T(t-s) f\left(s_{i}^{n}-\right)$, where $s_{i-1}^{n}<s \leqslant s_{i}^{n}, i=1, \ldots, n$, and $g_{n}(0)=T(t) f(0)$. By $(2.4),\left\{g_{n}\right\}$ is bounded on $[0, t]$ and $\left\{g_{n}\right\}$ converges to $T(t-s) f(s)$ almost everywhere on $[0, t]$. By the Lebesgue theorem,

(3.3) $\lim _{n \rightarrow \infty} \int_{0}^{t} g_{n}(s) d s=\lim _{n \rightarrow \infty} \sum_{i=1}^{n} \int_{s_{i-1}^{n}}^{s_{i}^{n}} T(t-s) f\left(s_{i}^{n}-\right) d s=\int_{0}^{t} T(t-s) f(s) d s$ (see [3, Theorem 3.7 .9 , p. 83]). From [4, p. 486], $\int_{0}^{t} g_{n}(s) d s \in D(A)$ and

$$
A \int_{0}^{t} g_{n}(s) d s=\sum_{i=1}^{n}\left(T\left(t-s_{i}^{n}\right)-T\left(t-s_{i-1}^{n}\right)\right) f\left(s_{i}^{n}-\right) .
$$

Then, by (3.2), (3.3), (3.4), and the closedness of $A$ we obtain (3.1).

LEMMA 3.2. $A \int_{0}^{t} T(t-s) f(s) d s$ is continuous from the right in $t$ on $[0, r)$.

'Proof. Let $0 \leqslant t<r$. First, we show that

$$
\lim _{h \rightarrow 0^{+}} A \int_{t}^{t+h} T(t+h-s) f(s) d s=0 .
$$

We observe that an argument similar to that of Lemma 3.1 shows that 


$$
\int_{t}^{t+h} T(t+h-s) f(s) d s \in D(A)
$$

and

$$
A \int_{t}^{t+h} T(t+h-s) f(s) d s=\int_{t}^{t+h} d T(t+h-s) f(s-) .
$$

Take $h>0$ and sufficiently small. If $\varepsilon>0$ there is a chain $\left\{s_{i}\right\}_{i=0}^{n}$ from $t$ to $t+h$ such that

$$
\begin{aligned}
\| \int_{t}^{t+h}(T(h) & -T(t+h-s)) d f(s-) \| \\
& <\left\|\sum_{i=1}^{n}\left(T(h)-T\left(t+h-s_{i-1}\right)\right)\left(f\left(s_{i}-\right)-f\left(s_{i-1}-\right)\right)\right\|+\varepsilon \\
& <2 M \sum_{i=2}^{n}\left\|f\left(s_{i}-\right)-f\left(s_{i-1}-\right)\right\|+\varepsilon \\
& <2 M\left(\nu(t+h)-\nu\left(s_{1}\right)\right)+\varepsilon .
\end{aligned}
$$

Then, (3.6) yields

$$
\begin{aligned}
\| \int_{t}^{t+h}(T(h) & -T(t+h-s)) d f(s-) \| \\
& \leqslant 2 M\left(\nu(t+h)-\lim _{s \rightarrow t^{+}} \nu(s)\right) .
\end{aligned}
$$

An integration by parts (see [2, Theorem 11.7, p. 53]) together with (3.7) yields

$$
\begin{aligned}
& \left\|A \int_{t}^{t+h} T(t+h-s) f(s) d s\right\|=\left\|\int_{t}^{t+h} d T(t+h-s) f(s-)\right\| \\
& =\left\|-\int_{t}^{t+h} T(t+h-s) d f(s-)+f((t+h)-)-T(h) f(t-)\right\| \\
& \begin{aligned}
(3.8) \quad=\| \int_{t}^{t+h}(T(h)-T(t+h-s)) d f(s-) \\
\quad+f((t+h)-)-T(h) f((t+h)-) \|
\end{aligned} \\
& \quad<2 M\left(\nu(t+h)-\lim _{s \rightarrow t^{+}} \nu(s)\right)+\|(I-T(h)) f((t+h)-)\| .
\end{aligned}
$$

In order to establish (3.5) we need only show that

$$
\lim _{h \rightarrow 0^{+}}\|(I-T(h)) f((t+h)-)\|=0 .
$$

But (3.9) holds by virtue of the fact that the range of $f(\cdot-)$ on $[0, r]$ lies in a compact set of $X$ and $\lim _{h \rightarrow 0^{+}}(I-T(h)) z=0$ uniformly for $z$ in a compact set. The right continuity of $A \int_{0}^{t} T(t-s) f(s) d s$ in $t$ now follows from (3.5) and the fact that 


$$
\begin{aligned}
A \int_{0}^{t+h} T(t & +h-s) f(s) d s-A \int_{0}^{t} T(t-s) f(s) d s \\
& =(T(h)-I) A \int_{0}^{t} T(t-s) f(s) d s+A \int_{t}^{t+h} T(t+h-s) f(s) d s .
\end{aligned}
$$

LEMMA 3.3. $A \int_{0}^{t} T(t-s) f(s) d s$ is continuous from the left in $t$ on $(0, r]$.

Proof. Let $0<t \leqslant r$. Observe that for $c>0$ and sufficiently small,

$$
\begin{aligned}
\left\|A \int_{t-c}^{t} T(t-s) f(s) d s\right\|=\left\|\int_{t-c}^{t} d T(t-s) f(s-)\right\| \\
=\left\|-\int_{t-c}^{t} T(t-s) d f(s-)+f(t-)-T(c) f((t-c)-)\right\| \\
<M(\nu(t)-\nu(t-c))+M\|f(t-)-f((t-c)-)\| \\
\quad+\|(I-T(c)) f(t-)\| .
\end{aligned}
$$

If $h>0$ and $c>0$ are both sufficiently small, then (3.10) applied twice below yields

$$
\begin{aligned}
& \left\|A \int_{0}^{t} T(t-s) f(s) d s-A \int_{0}^{t-h} T(t-h-s) f(s) d s\right\| \\
& =\| A T(c)\left(\int_{0}^{t-h-c}(T(h)-I) T(t-h-c-s) f(s) d s\right. \\
& \left.+\int_{t-h-c}^{t-c} T(t-c-s) f(s) d s\right) \\
& +A \int_{t-c}^{t} T(t-s) f(s) d s-A \int_{t-h-c}^{t-h} T(t-h-s) f(s) d s \| \\
& \leqslant|A T(c)|\left(\left\|\int_{0}^{t-h-c}(T(h)-I) T(t-h-c-s) f(s) d s\right\|\right. \\
& \left.+M h \sup _{s \in[t-h-c, t-c]}\|f(s)\|\right) \\
& +M(\nu(t)-\nu(t-c))+M\|f(t-)-f((t-c)-)\| \\
& +\|(I-T(c)) f(t-)\|+M(\nu(t-h)-\nu(t-h-c)) \\
& +M\|f((t-h)-)-f((t-h-c)-)\| \\
& +\|(I-T(c)) f((t-h)-)\| \text {. }
\end{aligned}
$$

For a given $\varepsilon>0$ first choose $c>0$ and then choose $\delta>0$ such that if $0<h<\delta$, then (3.11) is $<\varepsilon$ (use the fact that $\nu$ and $f(\cdot-)$ are left continuous at $t$ and $\lim _{h \rightarrow 0^{+}}(T(h)-I) z=0$ uniformly for $z$ in a compact set). The left continuity of $A \int_{0}^{t} T(t-s) f(s) d s$ then follows immediately.

To complete the proof of the theorem we see that (1.3) follows from 
Lemmas 3.1, 3.2, and 3.3. To prove (1.4) let $0<t<r$ and observe that for $h>0$ and sufficiently small we have

$$
\begin{aligned}
(u(t+h)-u(t)) / h= & (T(t+h) x-T(t) x) / h \\
& +\frac{1}{h} \int_{t}^{t+h} T(t+h-s) f(s) d s \\
& +\frac{T(h)-I}{h} \int_{0}^{t} T(t-s) f(s) d s .
\end{aligned}
$$

By (2.1)

$$
\lim _{h \rightarrow 0^{+}} \frac{1}{h} \int_{t}^{t+h} T(t+h-s) f(s) d s=f(t+)
$$

and (1.4) then follows from Lemmas 3.1 and 3.2. To prove (1.5) let $0<t \leqslant r$ and observe that for $h>0$ and sufficiently small we have

$$
\begin{aligned}
(u(t-h)-u(t)) /(-h)= & (T(t-h) x-T(t) x) /(-h) \\
& +\frac{1}{h} \int_{t-h}^{t} T(t-s) f(s) d s \\
& +\frac{T(h)-I}{h} \int_{0}^{t-h} T(t-h-s) f(s) d s .
\end{aligned}
$$

By (2.2)

$$
\lim _{h \rightarrow 0^{+}} \frac{1}{h} \int_{t-h}^{t} T(t-h-s) f(s) d s=f(t-)
$$

Denote

$$
z(h) \stackrel{\text { def }}{=} \int_{0}^{t-h} T(t-h-s) f(s) d s .
$$

By Lemma 3.1, $z(h) \in D(A)$ and by Lemma 3.3,

$$
\lim _{h \rightarrow 0^{+}} \frac{T(h)-I}{h} z(h)=\lim _{h \rightarrow 0^{+}} \frac{1}{h} \int_{0}^{h} T(s) A z(h) d s=A z(0),
$$

which yields (1.5). Finally, (1.6) follows immediately from (1.4), (1.5), and (2.4).

We conclude with the observation that our theorem may be applied to nonlinear evolution equations of the form $d u(t) / d t=A u(t)+B(u(t))$. If $-B$ is an accretive continuous everywhere defined nonlinear operator on $X$, then there exists a solution $u(t)$ to the Volterra integral equation

$$
u(t)=T(t) x+\int_{0}^{t} T(t-s) B(u(s)) d s, \quad x \in X
$$

(see [10, Theorem I]). If we assume that $x \in D(A)$, then it can be shown that $u(t)$ is Lipschitz continuous. If we also assume that $B$ is Lipschitz continuous and $T(t) X \subset D(A)$ for all $t>0$, then our theorem implies $u(t)$ satisfies $d u(t) / d t=A u(t)+B(u(t))$ for all $t \geqslant 0$. If it is not true that $T(t) X \subset$ $D(A)$, then this conclusion may not hold (see [10, Example 4.1]). A similar 
observation is made in [7] for the case that $T(t), t \geqslant 0$, is a holomorphic semigroup.

\section{REFERENCES}

1. P. Benilan, Opérateurs m-accrétifs hémicontinus dans un espace de Banach quelconque, $\mathbf{C} . \mathbf{R}$. Acad. Sci. Paris Sér. A 278 (1974), 1029-1032. MR 50 \# 1064.

2. T. H. Hildebrandt, Introduction to the theory of integration, Academic Press, New York, 1963. MR 27 \#4900.

3. E. Hille and R. S. Phillips, Functional analysis and semi-groups, rev. ed., Amer. Math. Soc. Colloq. Publ., vol. 31, Amer. Math. Soc., Providence, R. I., 1957. MR 19, 664.

4. T. Kato, Perturbation theory for linear operators, Springer-Verlag, New York, 1966. MR 34 \#3324.

5. S. G. Krein, Linear differential equations in Banach space, "Nauka", Moscow, 1967; English transl., Transl. Math. Monographs, vol. 29, Amer. Math. Soc., Providence, R. I., 1972. MR 40 \# 508.

6. A. Pazy, Approximation of the identity operator by semigroups of linear operators, Proc. Amer. Math. Soc. 30 (1971), 147-150. MR 44 \#4568.

7. _,$A$ class of semi-linear equations of evolution (to appear).

8. R. S. Phillips, Perturbation theory for semi-groups of linear operators, Trans. Amer. Math. Soc. 74 (1953), 199-221. MR 14, 882.

9. B. Sz.-Nagy, Introduction to real functions and orthogonal expansions, Oxford Univ. Press, New York, 1965. MR 31 \#5938.

10. G. F. Webb, Continuous nonlinear perturbations of linear accretive operators in Banach spaces, J. Functional Analysis 10 (1972), 191-203. MR 50 \# 14407.

Department of Mathematics, Vanderbilt University, Nashville, Tennessee 37235 